\title{
Probing the heliosphere with the directional anisotropy of galactic cosmic-ray intensity
}

\author{
Kazuoki Munakata ${ }^{1}$ \\ ${ }^{1}$ Physics Department, Shinshu University, \\ 3-1-1 Asahi, Matsumoto, Nagano 390-8621, Japan \\ email: kmuna00@shinshu-u.ac.jp
}

\begin{abstract}
Because of the large detector volume that can be deployed, ground-based detectors remain state-of-the-art instrumentation for measuring high-energy galactic cosmic-rays (GCRs). This paper demonstrates how useful information can be derived from observations of the directional anisotropy of the high-energy GCR intensity, introducing the most recent results obtained from the ground-based observations. The anisotropy observed with the global muon detector network (GMDN) provides us with a unique information of the spatial gradient of the GCR density which reflects the large-scale magnetic structure in the heliosphere. The solar cycle variation of the gradient gives an important information on the GCR transport in the heliosphere, while the short-term variation of the gradient enables us to deduce the large-scale geometry of the magnetic flux rope and the interplanetary coronal mass ejection (ICME). Real-time monitoring of the precursory anisotropy which has often been observed at the Earth preceding the arrival of the ICME accompanied by a strong shock may provide us with useful tools for forecasting the space weather with a long lead time. The solar cycle variation of the Sun's shadow observed in the $\mathrm{TeV}$ GCR intensity is also useful for probing the large-scale magnetic structure of the solar corona.
\end{abstract}

Keywords. Galactic cosmic-rays, Cosmic-ray anisotropy, Cosmic-ray density gradient, Cosmicray precursors of ICME, Sun's shadow in $10 \mathrm{TeV}$ cosmic-ray intensity

\section{Introduction}

Galactic cosmic-rays (GCRs) are extremely high-energy nuclei that travel close to the speed of light in space. They are ubiquitous in the Milky Way and make up a substantial fraction of the total energy of the Galaxy, equivalent to the energy in large-scale magnetic fields and thermal gases. Being charged particles, they are deflected when crossing the magnetic field in the space, and the amount of the total deflection in an average magnetic field magnitude is dependent on both their momentum and path lengths. The cosmic-ray flux at energies high enough to undergo minimal deflection is so small that cosmic-ray sources in the Galaxy far away from us have been proved difficult to be observed directly by measuring the directional anisotropy of GCR intensity. The significant deflection and the pitch angle scattering by the irregular magnetic field, on the other hand, produce the diffusive streaming of GCRs which has been observed as a GCR anisotropy at the Earth with an amplitude of $\sim 0.1-1.0 \%$. The present paper demonstrates how useful information can be derived from the ground-based observations of the anisotropy.

Ground-based detectors of GCRs use the atmosphere as an active component and measure secondary particles produced from the interaction between the atmospheric nuclei and the primary cosmic-rays which are mostly protons. Because of the large detector volume that can be deployed at ground-based stations, neutron monitors (Simpson, Fonger \& Treiman 1953) and muon detectors (Fujimoto et al. 1984) remain state-of-the-art instrumentation for measuring $>1 \mathrm{GeV}$ cosmic-rays. These instruments excel at recording 
time variations of the cosmic-ray flux with a great statistical accuracy which is high enough for measuring the typically small directional anisotropy. Their energy range is highly complementary with the upper range of energies measured by cosmic-ray detectors flown in space.

There are several reasons that particles at these energies are interesting from a space weather perspective. First, they travel nearly at the speed of light. Cosmic-ray particles that interact with a shock or the interplanetary coronal mass ejection (ICME) and escape into the upstream region will race ahead of the much slower shock, bringing advance warning of a disturbance approaching the Earth. Second, the particles have large mean free paths of the pitch angle scattering in the irregular magnetic field. This is important because precursory signatures of an approaching disturbance will be wiped out by scattering on scales larger than a mean free path. Third, the particles have Larmor radii that are large compared to Earth's magnetosphere, but are smaller than or comparable to the scale size of a typical disturbance. For instance, a typical energy of primary GCRs for neutron monitors would be $10 \mathrm{GeV}$ which corresponds to a Larmor radius of $0.02 \mathrm{AU}$ in a $10 \mathrm{nT}$ field. A typical energy for muon detectors is $50 \mathrm{GeV}$ which corresponds to a Larmor radius of $0.1 \mathrm{AU}$. This is significant because it implies that kinetic anisotropies, such as the diamagnetic drift anisotropy expressed by a product of the Larmor radius and the spatial gradient of the cosmic-ray density, are responding to the large-scale structure of the solar wind disturbance. Instrumentation and methods for using cosmic-rays in space weather applications have advanced dramatically in recent years. The existing muon detector network has been improved to consist of four multi-directional muon detectors in Japan (Nagoya), Australia (Hobart), Brazil (São Martinho) and Kuwait (Kuwait), now forming the Global Muon Detector Network (GMDN). For the detail information of the GMDN and data analyses, readers can refer to Okazaki et al. (2008). The most recent results derived from observations with the GMDN are introduced in the following sections 2 and 3, while the solar cycle variation of the Sun's shadow observed in an extremely high-energy GCR intensity is briefly reported in section 4.

\section{Observations of the spatial gradient of cosmic-ray density in the heliosphere}

The GCR intensity recorded at the Earth changes in the solar activity- and magneticcycles reflecting the solar cycle variations of the modulation parameters such as the sunspot number, the magnitude of the interplanetary magnetic field (IMF) and the tiltangle of the heliospheric current sheet (HCS). The best-known example of such variation is the 11-year variation of the count rate of neutron monitors which represents the temporal variation of the omnidirectional intensity of GCRs. While the omnidirectional intensity measured by a single detector represents the temporal variation of the GCR density at the single location of the detector, the directional anisotropy of the GCR intensity tells us about the spatial distribution of the GCR density around the detector. Since the magnitude of the GCR anisotropy due to the diffusive streaming is proportional to the spatial gradient of the GCR density, one can derive the gradient vector in three dimensions from the anisotropy precisely observed with the global network of detectors. The spatial density gradient is important, because it tells us the average feature of the large-scale magnetic field which is governing the spatial distribution of GCRs in the heliosphere and is still difficult to derive directly from any other in-situ and/or ground-based measurements.

An example of such observations by the GMDN is shown in Fig. 1 which displays the solar cycle variation of the GCR gradient since 2001 (Kozai et al. 2011). The negative 

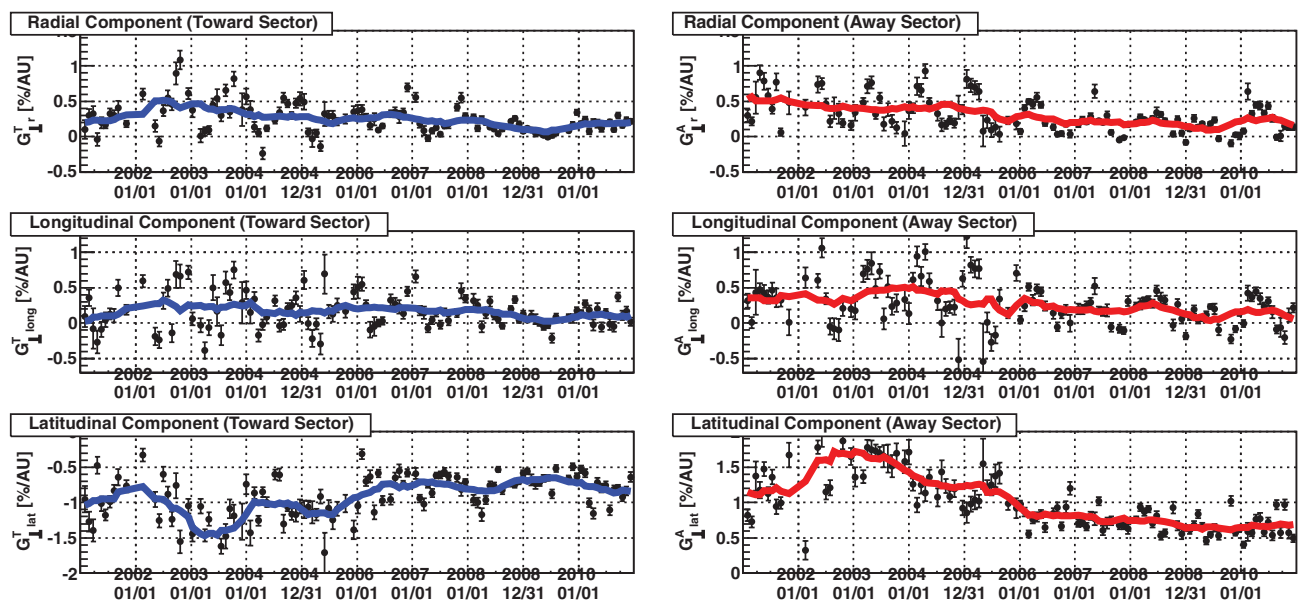

Figure 1. Derived density gradient perpendicular to the IMF. Solid circles in the left (right) panels display the average three components $(r, \theta, \phi)$ of $\boldsymbol{G}_{\perp}(t)$ in the heliocentric polar coordinate system in the Toward (Away) IMF sector in every rotation. The thick curve in each panel represents the central moving average of the solid circles over 14 rotations. From now on, colour figures refer to the online version of this paper.

(positive) latitudinal gradient $G_{\perp l a t}(t)$ in Toward (Away) IMF sector in the bottom panel of Fig. 1 indicates the local maximum of the GCR density on the HCS separating IMF sectors. This is qualitatively in accord with the "drift model" prediction for the density distribution during the "neagtive" polarity period of the solar polar magnetic field (also referred as the $A<0$ epoch) (Kóta \& Jokipii 1983). In Fig. 1, it is also clear that $\left|G_{\perp l a t}(t)\right|$ decreases significantly from 2003 to 2008-2009 according to the solar activity decrease. The long-term variation of the GCR gradient has attracted less attention than the 11-year variation of the neutron monitor's count rate, but it also provides us with an important information of the global distribution of GCRs reflecting the large-scale magnetic structure of the heliosphere.

Shown in Fig. 2 is another example of the GCR gradient observed in association with the arrival of the ICME accompanied by a strong interplanetary shock (Kuwabara et al. 2009). The best-fit density in the top left panel shows a clear signature of a large Forbush decrease (FD) indicating that the Earth entered in the GCR depleted region formed downstream of the shock and inside the ICME. Based on numerical simulations of the cross-field diffusion of GCRs into the magnetic flux rope which is modeled with an expanding straight "cylinder" as an idealized representation of a local section of a flux rope, the best-fitting analyses between the observed and model density gradients are performed and the cylinder geometry, represented by the best-fit parameters including the radius, the expansion speed and the orientation of the cylinder axis, is derived ( $\mathrm{Mu}-$ nakata et al. 2006, Kuwabara et al. 2004, 2009). The ICME structure in this event is also determined independently from a magnetic flux rope analyses of the $A C E$ (Advanced Composition Explorer) magnetic field and solar wind data and is compared with the one from the cosmic-ray analyses. It is seen in the bottom panels that two geometries are very consistent with each other. From March 2001 to May 2005, 11 ICME events that produced FDs $>2 \%$ were observed, and clear variations of the density gradient due to ICME passage were observed in 8 of 11 events. In five of the eight events, signatures of magnetic flux rope structure (large, smooth rotation of magnetic field) were also seen, and the ICME geometry and orientation deduced from the two methods were very 

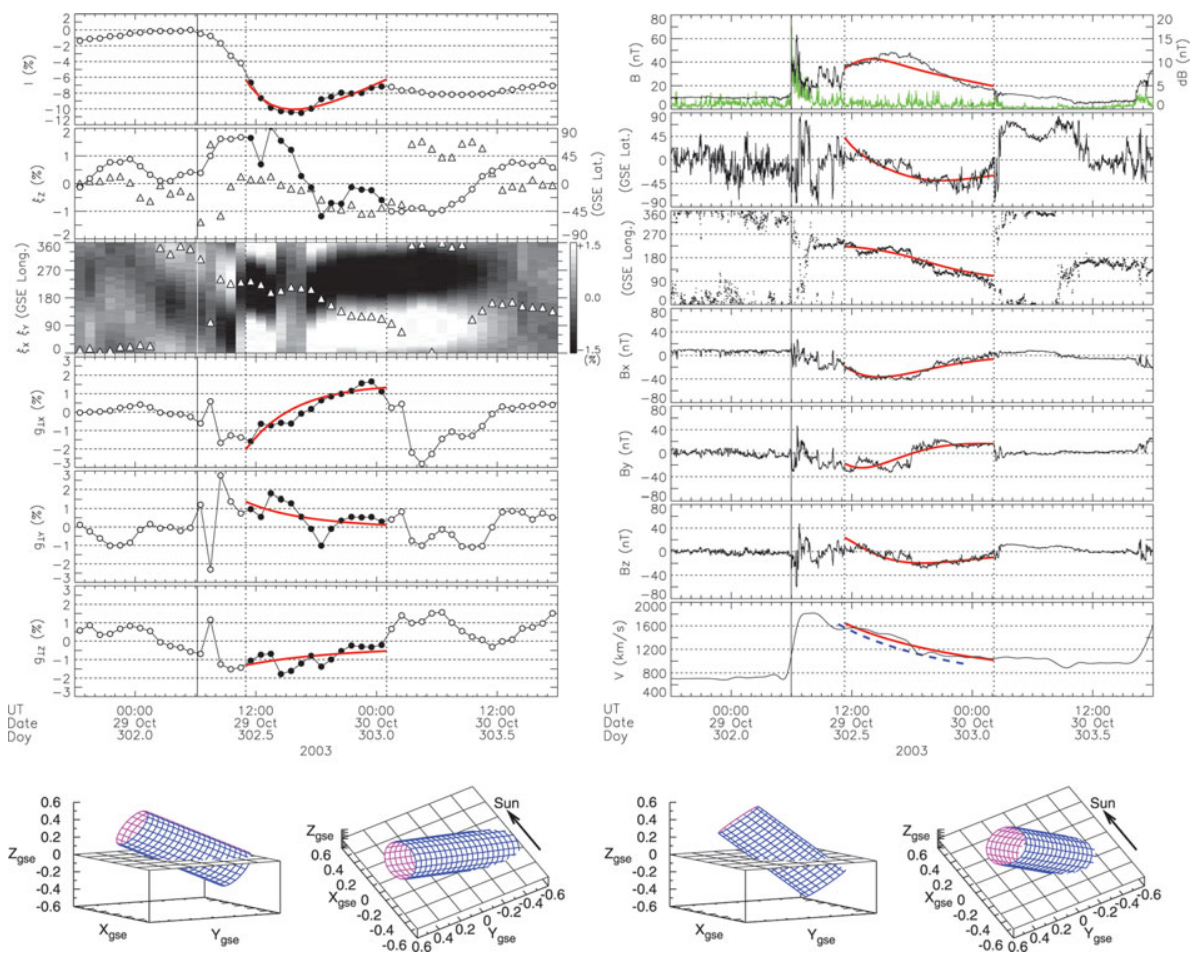

Figure 2. Observation and modeling of ICME geometry on 29 October 2003 (left) from cosmic-ray density gradients observed by the muon detector network and (right) from magnetic flux rope model based upon $A C E$ IMF measurements. Each left panel from the top shows hourly value of the observed GCR density, north-south anisotropy, the component anisotropy in the ecliptic plane in a gray scale format, and the three components of the density gradient in GSE coordinates. Latitude and longitude of the hourly mean IMF are plotted over the anisotropy as triangle marks in the second and third panels, respectively. The vertical solid line shows the arrival time of the shock, while the dotted lines show ICME/flux rope boundaries. In bottom panels, the cylinder geometry in GSE coordinates deduced from both methods is compared.

similar in three events. This suggests that the cosmic-ray based method can be used as a complementary method for deducing ICME geometry especially for events where a large FD is observed.

\section{Cosmic-ray precursors of the interplanetary disturbance}

While the relationship between ICMEs and FDs of cosmic-ray intensity is now well established (Cane 1993, Cane et al. 1994, 1996), it is less generally recognized that cosmicray decreases are often accompanied by strong enhancements of the cosmic-ray anisotropy (Lookwood 1971, Duggal \& Pomerantz 1976; Nagashima et al. 1992), some of which extend into the region upstream of the approaching shock. Such precursory anisotropies provide a key mechanism by which information about the presence of a disturbance can be transmitted to remote locations. Because cosmic-rays are fast and have large scattering mean free paths in the solar wind, this information is carried rapidly to the Earth and can be useful for space weather forecasting. Precursory anisotropies have generally been interpreted as kinetic effects related to interaction of ambient cosmicrays with the approaching shock (Barnden 1971; Nagashima et al. 1994; Belov et al. 2001; Leerungnavarat et al. 2003). Precursory decreases may result from a "loss-cone" 
(LC) effect, in which the station is magnetically connected to the cosmic-ray depleted region downstream of the shock. Precursory increases may result from particles that have received a small energy boost by reflecting from the approaching shock (Dorman et al. 1995). The LCs are typically visible at the Earth 4-8 hours ahead of the shock arrival associated with major geomagnetic storms (Munakata et al. 2000; Rockenbach et al. 2011).

An example of a LC precursor preceding a large geomagnetic disturbance in December 2006 is shown in Fig. 3 (Fushishita et al. 2010). This figure displays the intensity maps observed with the GMDN during 36 hours between the flare onset (02:14 UT, December $13)$ and the SSC (14:14 UT, December 14). Each square panel is the observation in one hour indicated by the day-of-year (DOY) above the panel. Each muon detector in the GMDN consists of two identical horizontal layers of unit detectors, vertically separated by a certain distance. By counting pulses of the twofold coincidences between a pair of detectors on the upper and lower layers, one can record the rate of muons from the corresponding incident direction. If one has a square $n \times n$ array of unit detectors aligned to the north-south (or east-west) direction in the $i$-th muon detector, therefore, one can record muon rates $I_{i(k, l)}^{o b s}(t)(k, l=-n+1, \ldots, 0, \ldots, n-1)$ in total $(2 n-1) \times$ $(2 n-1)$ directional channels at the time $t$, where positive (negative) $k$ and $l$ represent eastern (western) and northern (southern) inclined incident, respectively, with $k=l=0$ corresponding to the vertical incident. $I_{i(k, l)}^{o b s}(t)$ is divided by the statistical count error in each pixel and plotted as a function of $k$ and $l$ respectively on the horizontal and vertical axes in a color-coded format in each square panel of Fig. 3, which is called the "2D significance map" of the muon intensity. Panels in Fig. 3a, c and e display the 2D maps observed by São Martinho (Brazil), Hobart (Australia) and Kuwait (Kuwait), respectively. Red (blue) color in each pixel denotes the excess (deficit) intensity relative to the ominidirectional intensity in an entire field of view (FOV) of each detector. Also shown by white curves in each panel are contour lines of the pitch-angle measured from the sunward IMF direction and calculated for cosmic-rays incident to each pixel. It is seen that the zero pitch-angle region is first captured in the FOV of São Martinho in DOY 347.354-347.563 (08:00-13:00 UT, December 13), and then by Hobart in DOY 347.771347.979 (18:00-23:00 UT, December 13) and again by São Martinho in DOY 348.354348.563 (08:00-13:00 UT, December 14) according to the Earth's spin. A striking feature of this event is that a LC signature (i.e. intensity deficit around the zero pitch-angle) is seen first by São Martinho more than one day before the SSC. This suggests that the LC precursor already existed only 7 hours after the CME eruption at 02:54 UT on December 13 , when the interplanetary shock driven by an ICME was located at 0.4 AU from the Sun (Liu et al. 2008).

Based on a pitch angle distribution deduced from numerical simulations of high-energy particle transport across the shock (Leerungnavarat et al. 2003), the best-fit analyses to the data in Fig. 3 are carried out and the best-fit parameters denoting the model anisotropy are derived. The $2 \mathrm{D}$ maps reproduced from the best-fit parameters are shown in Figs. 3b, 3d and 3f. The best-fit analyses indicate that the lead time of this LC precursor is as long as 16 hours and the maximum intensity deficit at the LC center exceeds $-6 \%$ which is almost twice the size of the FD observed with the GMDN. This implies that the maximum intensity depression behind the shock is much larger than the FD size recorded at the Earth in this event. The precursor observed with the long lead time, like the event in Fig. 3, is of particular importance for the possible space weather forecast using cosmic-ray measurements. For an accurate observation of such event, however, one needs further improvement of the GMDN. First, an incomplete 
(a)

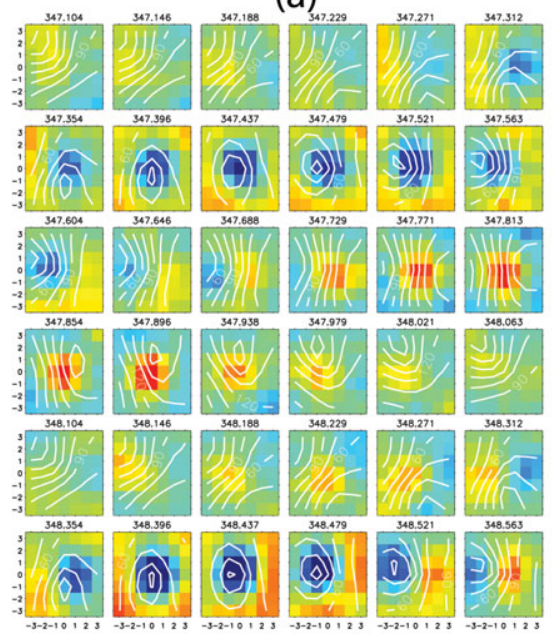

(c)

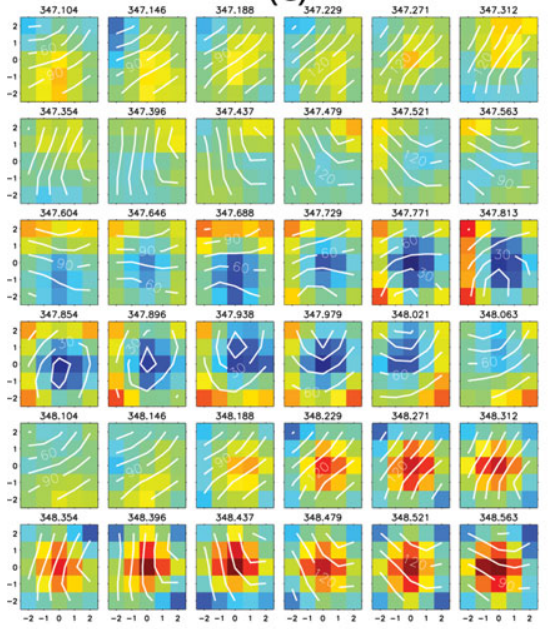

(e)

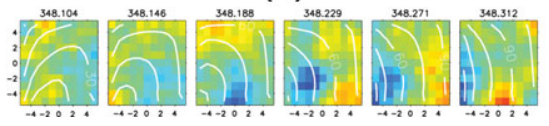

(b)

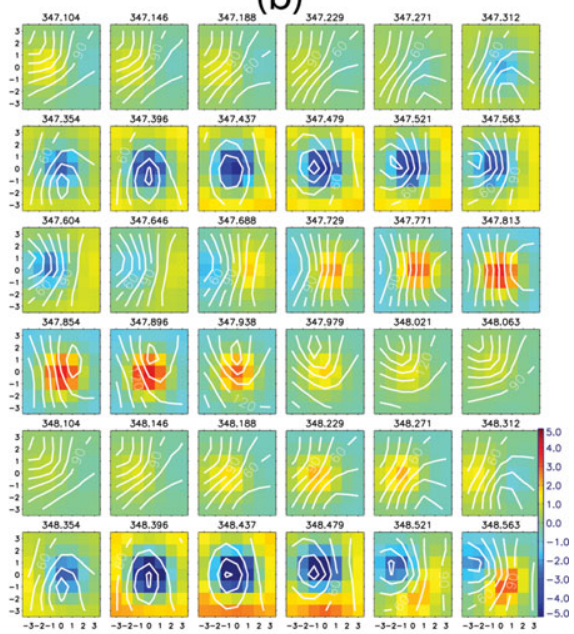

(d)
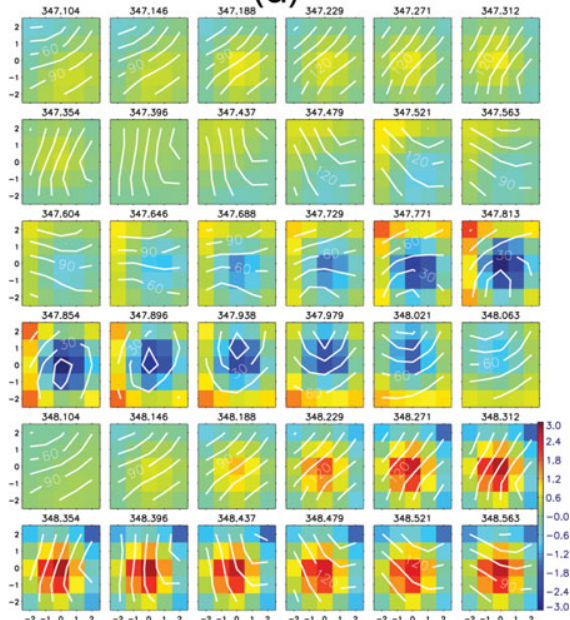

(f)

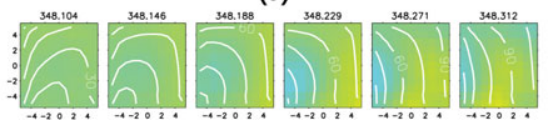

Figure 3. 2D significance maps observed by the GMDN prior to the SSC onset on December 14, 2006. The color scale is set \pm 5 (five times the statistical error) for São Martinho and \pm 3 (three times the statistical error) for Hobart and Kuwait as indicated by color bars at the right bottom corners of panels $\mathrm{b}$ and d. All maps during 36 hours between the flare onset and the SSC are shown in a-d for São Martinho and Hobart, while maps by Kuwait are shown only for 6 hours when the weak LC signature is visible with this small detector. The integers attached along the $\mathrm{x}$ - and $\mathrm{y}$-axes in each small panel indicate $k$ and $l$, representing respectively the east-west and north-south inclinations of the viewing direction (see text).

sky-coverage of the GMDN allowed us to analyze the LC anisotropy only for half a period, when the sunward IMF direction was in the FOV of the GMDN. Second, the insufficient detection areas in the network increased the statistical error and introduced a non-uniformity into the response of the GMDN to the LC anisotropy. Such a nonuniform response also introduced large fluctuations in the obtained best-fit parameters. 
These problems can be solved by expanding the detection area of the smallest detector in Kuwait and also by installing new detector(s) to expand the FOV of the GMDN, as a preparation for the next solar maximum expected at around the year of 2013 .

\section{Solar cycle variation of the Sun's shadow observed in $10 \mathrm{TeV}$ GCR intensity}

The anisotropy of GCRs with extremely high energies also provides us with unique information of the large-scale magnetic field of the Sun. The Larmor radius of a $10 \mathrm{TeV}$ (10 tera-electron-volt or $10^{13} \mathrm{eV}$ ) proton in $5 \mathrm{nT}$ IMF is as large as $40 \mathrm{AU}$ and GCRs with this energy travel nearly straight in the interplanetary space between the Sun and Earth. The Sun and Moon shield GCRs arriving from the directions behind them and cast tiny "shadows" in the GCR intensity observed at the Earth. The Sun's shadow is of particular interest because it reflects the large-scale solar magnetic field near the Sun, which is still difficult to observe with any direct and/or remote measurements. The Tibet Air Shower (AS) experiment at Yangbajing in Tibet, China has succeeded for the first time in observing a clear solar cycle variation of the Sun's shadow over an entire solar cycle. The Tibet AS experiment achieved the world highest count rate $(\sim 230 \mathrm{~Hz})$ and the best angular resolution $\left(\sim 0.9^{\circ}\right)$ of the GCR incident direction. For the Tibet AS experiment, readers can refer to Amenomori et al. (2009).

For analyzing the Sun's shadow, an on-source event number $\left(N_{\text {on }}\right)$ is first defined as the number of events arriving from the direction within a circle of $0.9^{\circ}$ radius from a certain point on the celestial sphere. The background or off-source event number $\left(N_{\text {off }}\right)$ is also calculated by averaging the number of events over the eight off-source windows which are located at the same zenith angle as the source direction but apart in the azimuthal direction. Both $N_{\text {on }}$ and $N_{\text {off }}$ are calculated on every $0.05^{\circ}$ grid of the Geocentric Solar Ecliptic (GSE) longitude and latitude surrounding the optical Sun's center. The deficit intensity relative to the background event number is then estimated as $D_{\text {obs }}=\left(N_{\text {on }}-\right.$ $\left.N_{\text {off }}\right) / N_{\text {off }}$ at every grid by using the yearly mean $N_{\text {on }}$ and $N_{\text {off }}$. Shown in Fig. 4 are 2D maps of $D_{\text {obs }}$ for every year from 1996 to 2009 (Amenomori et al. 2011). In each panel of this figure, $D_{\text {obs }}$ is plotted as a function of the GSE longitude and latitude on the horizontal and vertical axes, respectively. It is remarkable in this figure that $D_{\text {obs }}$ changes considerably in the solar activity cycle, i.e. the shadow becomes clear (with larger negative $D_{\text {obs }}$ ) around 1996 and 2008 when the solar activity is close to the minimum, while it becomes faint (with smaller negative $D_{\text {obs }}$ ) around 2000 when the activity was high. Since the Moon's shadow observed with the same apparatus during the same period is quite constant (Amenomori et al. 2009), the variation of the Sun's shadow in this figure is not likely of the instrumental origin.

For quantitative analyses of the temporal variation in Fig. 4 , the average $\left(\bar{D}_{\text {obs }}\right)$ of $D_{\text {obs }}$ within an on-source window around the origin of $2 \mathrm{D}$ map is calculated for every year. Fig. $5 \mathrm{c}$ shows the temporal variation of $\bar{D}_{\text {obs }}$ together with the sunspot number and the tilt-angle of the HCS (Wilcox Solar Observatory 2010). It is seen in Fig. $5 \mathrm{c}$ that $\bar{D}_{\text {obs }}$ shows a clear solar cycle variation with an amplitude as large as $50 \%$ of $\bar{D}_{\text {opt }}$ displayed by the horizontal line, which represents the deficit intensity expected when all cosmicrays from the direction of the optical Sun disk are excluded from the observation. Fig. 5 shows a good correlation between the variations of $\bar{D}_{\text {obs }}$ and the sunspot number or the HCS tilt-angle with the absolute correlation coefficient $(|r|)$ exceeding 0.85 . To examine the central position of the Sun's shadow, $D_{\text {obs }}$ in Fig. 4 are projected onto the horizontal and vertical axes and then the central GSE longitude and latitude of the projection are obtained for every year. In the average GSE latitude, no significant deviation from 

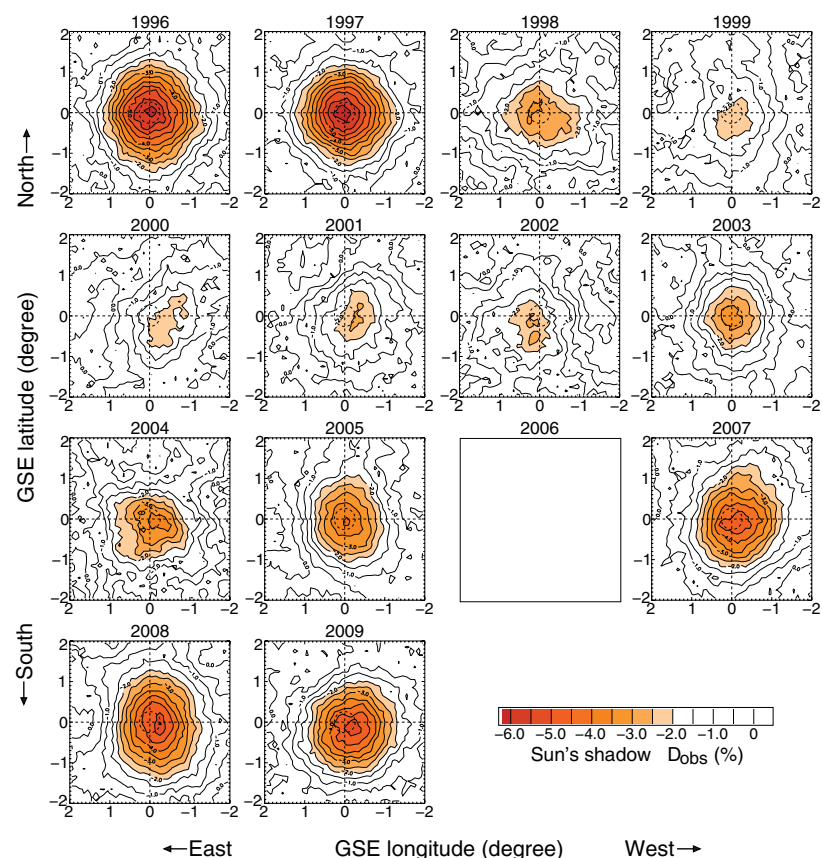

Sun's shadow Dobs $(\%)$

$\leftarrow$ East

GSE longitude (degree)

West $\rightarrow$

Figure 4. 2D maps of the observed Sun's shadow for every year between 1996 and 2009. Each panel displays a contour map of the yearly mean deficit intensity $\left(D_{\text {obs }}\right)$ as a function of the GSE longitude and latitude on the horizontal and vertical axes, respectively (see text). The data in 2006 are excluded from the plot since the AS array was scarcely operating due to upgrading the array and also due to instrumental troubles.

$0.0^{\circ}$ is found. The average GSE longitude in $1996-1997$ is also only $+0.039^{\circ} \pm 0.038^{\circ}$ which is significantly smaller than the observed geomagnetic deflection of the Moon's shadow $\left(-0.120^{\circ} \pm 0.024^{\circ}\right)$ (Amenomori et al. 2009). The average longitude in 20072009 during the next solar minimum period, on the other hand, is $-0.149^{\circ} \pm 0.036^{\circ}$ and significantly different from the longitude during the previous minimum in 1996-1997. This is due to the reversal of the solar dipole field during the solar maximum period around 2000. The magnetic deflection of the Sun's shadow in the solar corona is canceled by the geomagnetic deflection in the opposite sense in 1996-1997 solar minimum, while it is enhanced by the geomagnetic deflection in the same sense in 2007-2009 solar minimum. The detailed Monte Carlo (MC) simulations based on the coronal field model are now in progress aiming to clarify the physical implications of the observed solar cycle variation of the Sun's shadow. These simulations of the Sun's shadow enable us to examine coronal field model and probe the large-scale structure of the solar magnetic field as a function of time in the solar cycle.

\section{Acknowledgements}

The observations with the GMDN have been supported in part by Grants-in-Aid for Scientific Research from the Ministry of Education, Culture, Sports, Science and Technology in Japan, and by the joint research programs of the Solar-Terrestrial Environment Laboratory, Nagoya University. The observations with the Kuwait Muon Telescope are supported by the Kuwait University grant SP03/03. We thank N. F. Ness for providing the $A C E$ magnetic field data via the ACE Science Center. The collaborative experiment 

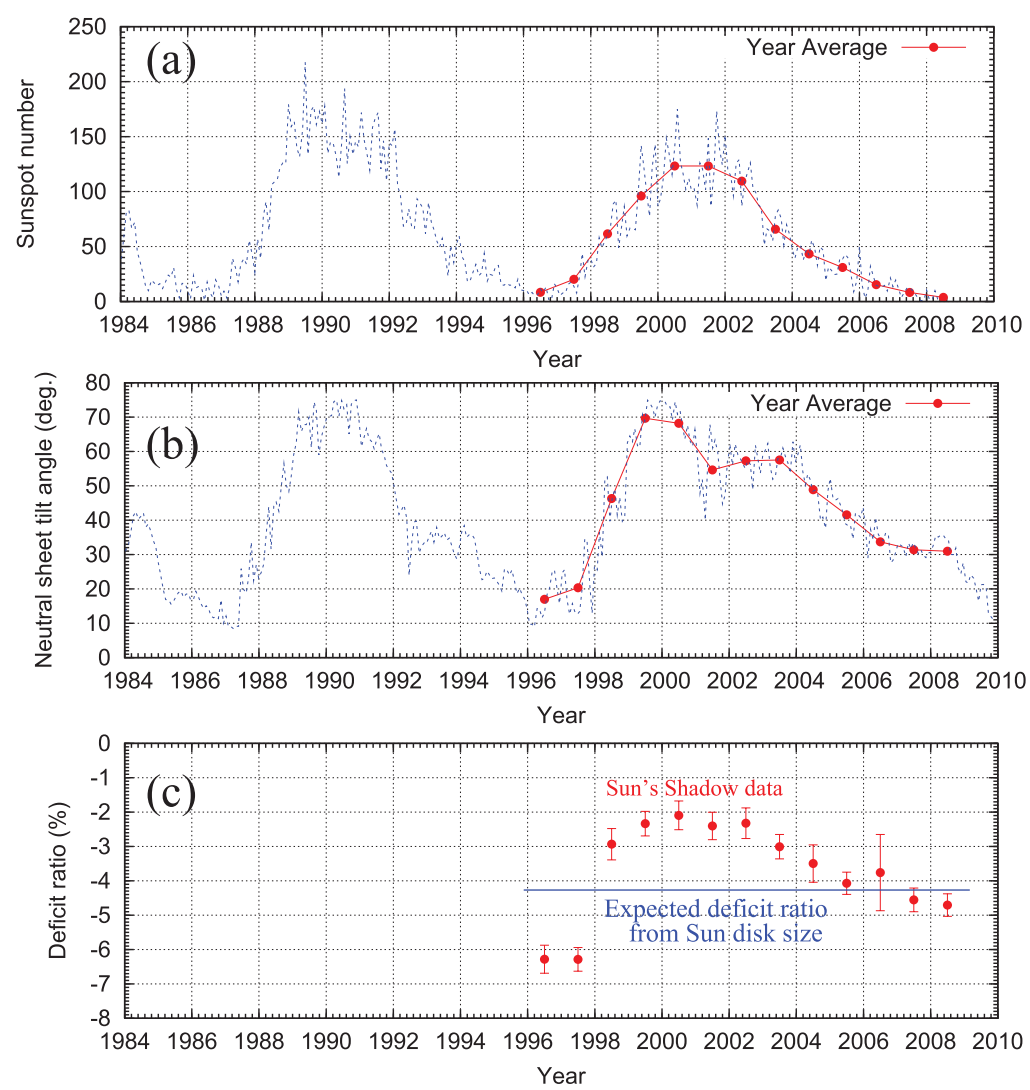

Figure 5. Solar cycle variations of the intensity deficit in the Sun's shadow. The solid circles in each panel from the top displays the yearly mean value of (a) the sunspot number, (b) the HCS tilt-angle in degree and, (c) yearly mean deficit intensity observed in the Sun's shadow $\left(\bar{D}_{\text {obs }}\right)$. The horizontal solid line in panel (c) indicates the deficit intensity expected when all cosmic-rays from the direction of the optical Sun disk are excluded from the observation.

of the Tibet Air Shower Arrays has been performed under the auspices of the Ministry of Science and Technology of China and the Ministry of Foreign Affairs of Japan. This work was supported in part by a Grant-in-Aid for Scientific Research on Priority Areas from the Ministry of Education, Culture, Sports, Science and Technology, by Grants-in-Aid for Science Research from the Japan Society for the Promotion of Science in Japan, and by the Grants from the National Natural Science Foundation of China and the Chinese Academy of Sciences.

\section{References}

Amenomori, M., Bi, X. J., Chen, D., Cui, S. W., \& Danzengluobu, Ding, L. K. et al. 2009, ApJ, 692,61

Amenomori, M., Bi, X. J., Chen, D., Chen, W. Y., \& Cui, S. W., Danzengluobu et al. 2011, Proc. of the 32nd Internat. Cosmic Ray Conf. (Beijing), 11, 242

Barnden, L. R. 1971, Solar Phys., 18, 165

Belov, A. V., Bieber, J. B., Eroshenko, E. A., Evenson, P., Pyle, R., \& Yanke, V. G. et al. 2001, Proc. of the 27th Internat. Cosmic Ray Conf. (Hamburg) (Schaltungsdienst Lange o.H.G., Berlin), 9, 3507

Cane, H. V. 1993, J. Geophys. Res., 98, 3509 
Cane, H. V., Richardson, I. G., von Rosenvinge, T. T., \& Wibberenz, G. 1994, J. Geophys. Res., 99, 21429

Cane, H. V., Richardson, I. G., \& von Rosenvinge, T. T. 1996, J. Geophys. Res., 101, 21561

Dorman, L. I., Iucci, N., \& Villoresi, G. 1995 Proc. of the 24th Internat. Cosmic Ray Conf. (Rome), 4, 892

Duggal, S. P. \& Pomerantz, M. A. 1976, J. Geophys. Res., 81, 5032

Fjimoto, K., Inoue, A., Murakami, K., \& Nagashima, K. 1984, Report of Cosmic-Ray Research Lab., No.9, Nagoya University

Fushishita, A., Kuwabara, T., Kato, C., Yasue, S., Bieber, J. W., \& Evenson, P. et al. 2010, ApJ, 715, 1239

Kóta, J. \& Jokipii, J. R. 1983, ApJ, 265, 573

Kozai, M., Munakata, K., Kato, C., Yasue, S., Kuwabara, T., \& Bieber, J. W. et al. 2011, Proc. of the 32nd Internat. Cosmic Ray Conf. (Beijing), 11, 301

Kuwabara, T., Munakata, K., Yasue, S., Kato, C., Akahane, S., \& Koyama, M. et al. 2004, Geophys. Res. Lett., 31, L19803-1

Kuwabara, T., Bieber, J. W., Evenson, P., Munakata, K., Yasue, S., \& Kato, C. et al. 2009, J. Geophys. Res., 114, A05109-1

Leerungnavarat, K., Ruffolo, D., \& Bieber, J. W. 2003, ApJ, 593, 587

Liu, Y., Luhmann, J. G., Müller-Mellin, R., Schroeder, P. C., Wang, L., \& Lin, R. P. et al. 2008, ApJ, 689, 563

Lookwood, J. A. 1971, Space Sci. Revs, 12, 658

Munakata, K., Bieber, J. W., Yasue, S., Kato, C., Koyama, M., \& Akahane, S. et al. 2000, J. Geophys. Res., 105, 27457

Munakata, K., Yasue, S., Kato, C., Kóta, J., Tokumaru, M., \& Kojima, M. et al. 2006, Adv. Geosci., 2, 115

Nagashima, K., Fujimoto, K., Sakakibara, S., Morishita, I., \& Tatsuoka, R. 1992, Planet. Space Sci., 40, 1109

Okazaki, Y., Fushishita, A., Narumi, T., Kato, C., Yasue, S., \& Kuwabara, T. et al. 2008, ApJ, 681,693

Rockenbach, M., Dal Lago, A., Gonzalez, W. D., Munakata, K., Kato, C., \& Kuwabara, T. et al. 2011, Geophys. Res. Lett., 38, L16108-1

Simpson, J. A., Fonger, W., \& Treiman, S. B. 1953, Phys. Rev., 90, 934

Wilcox Solar Observatory 2010, WSO Computed "Tilt Angle" of the Heliospheric Current Sheet, http://wso.stanford.edu/Tilts.html

\section{Discussion}

RAMÓN LóPEz: For this Sun shadow variation with the solar cycle, is the main driver the variation of the solar magnetic field?

Kazuoki Munakata: Yes, the strong magnetic field near the Sun has a major contribution in producing the Sun's shadow, we think.

DAVID WEBB: What is the energy of the particles in the shadow modeling?

Kazuoki Munakata: It is $10 \mathrm{TeV}$. 